\section{¿El contexto geográfico importa en la mortalidad por diabetes mellitus? Tendencias espacio-temporales en Argentina, 1990-2012}

\author{
Does geographic context matter in diabetes- \\ related mortality? Spatial and time trends in \\ Argentina, 1990-2012 \\ É importante o contexto geográfico na \\ mortalidadepor diabetes mellitus? Tendências \\ espaço-temporais na Argentina, 1990-2012
}

\section{Resumen}

El objetivo de esta investigación fue identificar conglomerados espacio-temporales de alta y baja mortalidad por diabetes mellitus durante el período 1990-2012 en Argentina. Se realizó un estudio ecológico retrospectivo espacio-temporal. Se consideró la población mayor de 34 años residente en Argentina, según sexo, durante el período 1990-2012. Las unidades espaciales de análisis estuvieron conformadas por departamentos más la Ciudad Autónoma de Buenos Aires. Se empleó la técnica de exploración espacio-temporal para la detección de conglomerados de alta y baja mortalidad. Se encontraron áreas de elevada mortalidad por diabetes mellitus en el centro-oeste del pais, y zonas de baja mortalidad en la región costera de la provincia de Buenos Aires y Patagonia. Los conglomerados de alta mortalidad ocuparon un período comprendido entre 1995 y 2008; los de baja mortalidad se desplazaron hacia los años 2002-2012. El descenso reciente en la mortalidad por diabetes mellitus no fue geográficamente homogéneo, con una disminución marcada en el este de la Provincia de Buenos Aires y la Ciudad Autónoma de Buenos Aires.

Diabetes Mellitus; Mortalidad Prematura; Análisis por Conglomerados
Carlos M. Leveau 1

M. Jimena Marro 2

Valeria Alonso 2

Alicia E. B. Lawrynowicz 2

doi: 10.1590/0102-311X00169615

\author{
Correspondencia \\ C. M. Leveau \\ Alte. Brown 2420 1o A, Mar del Plata, Buenos Aires - 7600 \\ Argentina. \\ cmleveau@hotmail.com \\ 1 Instituto de Salud Colectiva, Universidad Nacional de Lanús, \\ Lanús, Argentina \\ 2 Instituto Nacional de Epidemiología "Juan H. Jara" \\ Administración Nacional de Laboratorios e Institutos de Salud \\ “Dr. Carlos G. Malbrán”, Buenos Aires, Argentina.
}




\section{Introducción}

La diabetes mellitus es una enfermedad de curso progresivo que se caracteriza por hiperglucemia persistente, producto de alteraciones del metabolismo de los hidratos de carbono, grasas y proteínas, resultantes de la disminución de la secreción o acción de la insulina. Se acompaña de lesiones micro y macro vasculares, con consecuencias incapacitantes y riesgo aumentado de muerte prematura. La enfermedad se clasifica en dos tipos principales que responden a etiologías diferentes: la diabetes mellitus tipo I, de aparición más frecuente durante la infancia o adolescencia, y la tipo II (diabetes mellitus 2), responsable por alrededor del $90 \%$ de los casos de diabetes, es de presentación habitual en la edad adulta y se relaciona con factores de riesgo tales como obesidad e inactividad física 1.

A nivel mundial, aproximadamente 5,1 millones de personas con edades entre 20 y 79 años fallecieron a causa de diabetes en 2013, lo cual representó el 8,4\% de todas las causas de mortalidad en ese grupo etario; alrededor de la mitad de las defunciones ocurrieron en personas menores de 60 años, con escasa diferencia entre ambos sexos. En Sudamérica y América Central, en el año 2013, el 11,6\% de las muertes de la región se debieron a diabetes mellitus, lo que representó en términos absolutos unas 226.000 defunciones; un $53 \%$ de ellas aconteció en varones 2.

La tasa de mortalidad por diabetes mellitus en Argentina fue para el año 2012 de 19,33 casos por 100.000 habitantes; la distribución por sexos fue de 20,47 para hombres y 18,19 para mujeres, ocupando el séptimo lugar en el grupo de las principales causas de muerte para la población de nuestro país y el quinto en la franja etaria de 55 a 74 años 3 .

La evolución de la tasa de mortalidad ajustada por diabetes mellitus en Argentina entre 1997 y 2006 muestra las tasas más elevadas en el período 1999-2001, con valores de alrededor de 24 eventos por 100.000 habitantes, descendiendo luego de manera gradual hasta alcanzar los valores del inicio del período en 2006. Las tasas sufren un proceso de masculinización a partir de 1997, que se profundiza después de 20034.

Un aspecto relevante en el abordaje de las enfermedades crónicas no transmisibles (ECNT), de las cuales la diabetes mellitus forma parte, es la consideración del impacto de la mortalidad en función de su prematuridad; es decir, el análisis de las defunciones según su ocurrencia a una edad inferior a la esperada para determinada población y período 5. Para establecer este límite de edad de muerte prematura suele usarse la esperanza de vida al nacer, debido a que la misma representa la edad que se espera pueda vivir una persona en determinado lugar bajo las condiciones de mortalidad imperantes; de esa manera, las muertes prematuras se refieren a defunciones que ocurren en individuos antes de cumplir la expectativa de vida. Un denominador de uso común que se desprende de este abordaje es el de años de vida perdidos por muerte prematura 6 .

En base a estas observaciones se presentan los siguientes interrogantes: jlas variaciones temporales en la mortalidad por diabetes mellitus han sido homogéneas a lo largo de todo el territorio argentino? En caso contrario, jestas variaciones espacio-temporales han sido diferentes en varones y mujeres? Por último, ¿existen diferencias en los patrones de distribución espacio-temporal entre muertes prematuras y esperadas por diabetes mellitus?

Desde el paradigma de la geografía cuantitativa, la Ley de Tobler plantea que "todo está relacionado, pero las cosas más cercanas están más relacionadas que las distantes" 7 (p. 236). Si bien esta ley se vincula al concepto de asociación espacial en geografía 8 , también puede emparentarse al concepto de similitud temporal, en donde un proceso particular influencia de manera similar las tasas de cambio en diferentes localizaciones 9 . El concepto de interacción espacio-temporal integra ambas dimensiones, espacio y tiempo, y es un fenómeno que puede estar presente en ausencia de concentración espacial o temporal de casos 10. La interacción espacio-temporal se manifiesta cuando se observa una concentración espacial y, al mismo tiempo, una concentración temporal de casos de mortalidad. Es decir, los casos de mortalidad que se encuentran próximos en el espacio tienden a encontrarse cerca en el tiempo 11. Es así como un conglomerado espacio-temporal de alta mortalidad puede definirse como un área con una concentración no aleatoria de casos de mortalidad en espacio y tiempo. En contraparte, un conglomerado espacio-temporal de baja mortalidad nos está indicando una presencia de casos menores a lo esperado, en un área y durante un tiempo determinado.

El análisis espacio-temporal cuantitativo de los fenómenos de salud permitiría describir estas variaciones en el caso de la diabetes mellitus en Argentina y abrir el camino para el planteo de nuevas 
hipótesis sobre los factores que contribuyen a dichos fenómenos. En particular, el análisis espacial por conglomerados (clusters) permite localizar áreas de alta y baja mortalidad, con el fin de mejorar la eficiencia y evaluación de las políticas públicas de prevención en salud.

La mayoría de los estudios dedicados a analizar la distribución espacial de la diabetes se han enfocado en la incidencia y prevalencia de la diabetes mellitus $112,13,14,15,16,17$, mientras que pocos estudios han analizado los patrones de distribución espacial de la enfermedad de la diabetes mellitus 2 18,19,20,21. Green et al. 19 demostraron que tasas altas de diabetes mellitus 2 se agrupaban significativamente en el espacio, de acuerdo con características étnicas, socioeconómicas, ambientales y de estilo de vida de la población de un área urbana de Canadá. De manera similar, Tsai et al. 18 registraron niveles positivos de autocorrelación espacial en la mortalidad por diabetes mellitus 2 en Taiwán. Por otro lado, en la ciudad de Botucatu (São Paulo, Brasil) los casos de diabetes mellitus 2 no tendieron a concentrarse espacialmente 20 . La mayoría de estos estudios fue llevada a cabo en unos pocos países desarrollados, por lo que, a nuestro conocimiento, el comportamiento espacio-temporal de la diabetes mellitus no ha sido estudiado para América Latina, y especialmente Argentina.

El propósito del presente trabajo consiste en generar información relacionada con la dimensión espacio-temporal de la diabetes mellitus en la Argentina contemporánea. El objetivo de este estudio es analizar conglomerados espacio-temporales de alta y baja mortalidad por diabetes mellitus en varones y mujeres, considerando muertes "prematuras" y "esperadas", durante el período 1990-2012.

\section{Materiales y métodos}

Se realizó un estudio retrospectivo de diseño ecológico con aplicación de la técnica de escaneado espacio-temporal, mediante el uso del programa SaTScan, versión 9.3 (http: \\www.satscan.org). La población considerada fue la de ambos sexos mayores de 34 años y residentes en Argentina, durante el período 1990-2012. Se eligió trabajar con este grupo etario a partir de la observación, durante los años del período de estudio, de alrededor de dicha edad comenzaba a aumentar de manera exponencial la tasa de mortalidad por diabetes mellitus en ambos sexos para Argentina 21.

Las unidades espaciales de análisis fueron los departamentos (denominados partidos en la Provincia de Buenos Aires) de la República Argentina, unidades jurisdiccionales de nivel local, más la Ciudad Autónoma de Buenos Aires $(\mathrm{n}=498)$. Con el fin de contar con las mismas unidades espaciales a lo largo del período de estudio, quedaron conformadas seis unidades espaciales compuestas por departamentos/partidos que durante el periodo de estudio modificaron su extensión territorial al ceder o anexar territorio, o debido a la creación de nuevos departamentos/partidos.

El estudio utilizó fuentes secundarias de información consistentes en las bases de datos de mortalidad correspondientes al período 1990-2012, aportadas por la Dirección de Estadísticas e Información de Salud (DEIS), dependiente del Ministerio de Salud de Argentina, y los censos nacionales de población y vivienda de los años 1991, 2001 y 2010 del Instituto Nacional de Estadística y Censos (INDEC). Las tasas de mortalidad se calcularon tomando como referencia la población residente en cada área, de acuerdo a datos poblacionales obtenidos de los censos nacionales y proyecciones poblaciones realizadas durante los períodos inter-censales. En el caso de los datos poblacionales de cada uno de los años censales, se consideró la población por grupos quinquenales de edad, ya que si bien en los censos de 2001 y 2010 se cuenta con datos de edad en años simples, no ocurre lo mismo con los datos aportados durante el censo de 1991.

Las variables seleccionadas fueron el año en el que se registra el fallecimiento o al que pertenece el dato poblacional censal, el departamento de residencia habitual de la persona fallecida o la población de referencia, y la edad en años al momento de morir o de la población de referencia. Se incluyeron personas de ambos sexos con edad mayor a 34 años. Todos los procesamientos fueron por sexo en las categorías varón y mujer.

Para la causa de muerte se asignó la causa básica de muerte tabulada en la base de defunciones, codificada de acuerdo a la Clasificación Estadística Internacional de Enfermedades y Problemas Relacionados con la Salud en sus 9a y 10a revisión (CIE-9 y CIE-10 respectivamente). Se seleccionaron los códigos correspondientes a diabetes mellitus: 250 de la CIE-9 y E10 a E14 de la CIE-10 23. 
En diez provincias no se contó con datos de mortalidad disponibles durante los primeros años del período de estudio, por lo que la DEIS aportó datos de mortalidad de años anteriores 24: en Santiago del Estero se utilizaron los datos de mortalidad del año 1985 durante el período 1990-1994; en Tierra del Fuego y Tucumán, datos de 1987 para los años 1990 y 1991; en Salta, datos de 1986 para el año 1990, datos de 1988 para 1991 y 1992, y datos de 1992 para el año 1993; en Santa Cruz se emplearon los datos de 1989 para el trienio 1990-1992; en La Pampa, datos de 1992 para el año 1991; en Entre Ríos, datos de 1991 para el año 1992 y datos de 1992 para el año 1993; en San Luis se utilizaron los datos de 1991 para el año 1992; en Chubut se emplearon los datos de 1992 para el año 1991; y en la Ciudad Autónoma de Buenos Aires se utilizaron los datos de 1992 para el año 1993.

Se consideró muerte prematura a la defunción acontecida a una edad menor a la esperanza de vida al nacer para el año, sexo y provincia de referencia del departamento/partido de residencia, y muerte esperada a la defunción ocurrida a una edad igual o mayor a la esperanza de vida al nacer para el año, sexo y provincia de referencia del departamento/partido de residencia. El límite superior para las muertes prematuras se ubicó entre los 64 y 69 años en el caso de los varones, y entre los 69 y 74 años entre las mujeres. Estos límites se ubican cercanos a los 74 años establecidos como límite superior arbitrario de muertes evitables en otros estudios llevados a cabo en países desarrollados 25,26,27. Al reconocer el carácter arbitrario de este límite superior, se ha planteado que la esperanza de vida al nacer puede servir como límite superior para las muertes prematuras 5 .

La esperanza de vida al nacer representa los años que se espera que una persona pueda vivir si se mantuvieran constantes las condiciones y tasas de mortalidad vigentes al momento considerado. Se tomó la esperanza de vida al nacer por sexo y provincia a la que pertenece cada departamento: para los años pertenecientes al período 1990-1995 se consideró el dato estimado para 1990-1992 (INDEC. Tablas abreviadas de mortalidad provinciales por edad y sexo (1990-1992). Buenos Aires, Serie Análisis Demográfico, 4. http://www.indec.mecon.ar/nuevaweb/cuadros/2/ind_demograf_5.xls), para los años comprendidos en el intervalo 1996-2005, el correspondiente a 2000-2001 (INDEC. Tablas abreviadas de mortalidad 2000-2001. Total País y Provincia. Documento de Trabajo del Programa Análisis Demográfico, 146. http://www.indec.mecon.ar/nuevaweb/cuadros/2/ind_demograf_5.xls), y para los años entre 2006 y 2012, se utilizó la estimación de esperanza de vida al nacer para 2010 (INDEC. Tabla completa de mortalidad de la Argentina por sexo.1990-1992. Buenos Aires, Serie Análisis Demográfico, 3. http://www.indec.mecon.ar/nuevaweb/cuadros/2/ind_demograf_5.xls). Se trabajó con este criterio con la intención de que la condición de prematuridad de cada evento de muerte estuviera definida en función del escenario de mortalidad esperable en esa área, definido por el valor de la esperanza de vida al nacer.

Las estimaciones de la población de referencia para los años inter-censales fueron basadas en la esperanza de vida al nacer a nivel provincial; se asumió una variación anual igual a la calculada para los grupos quinquenales, cercanos al año de corte de la esperanza de vida al nacer, entre dos períodos censales. Como ejemplo del cálculo de la población de referencia, tomamos un partido (unidad espacial) localizado en la Provincia de Buenos Aires. Si consideramos el cálculo de la población masculina de referencia para casos prematuros de mortalidad entre los años 1990-2012, se establece que esta población debe estar comprendida entre el grupo quinquenal de los 35-39 y los 65-69 años, debido a que la Esperanza de Vida al Nacer promedio fue de 70 años para esa provincia (1990-1992: 69 años; 2000-2001: 70 años; 2010: 72 años). Luego de calcular la población masculina de referencia para los años 1991, 2001 y 2010, se calculó una tasa de variación anual inter-censal, utilizando los datos de los años censales. Con esta tasa se estimó la población anual para los años intercensales 1990, 1992-2000, 2002-2009 y 2011-2012.

La técnica de escaneado espacio-temporal utilizada en este estudio consiste en el rastreo estadístico espacio-temporal que genera conglomerados primarios y secundarios, con exceso o déficit de mortalidad prematura o esperada por diabetes mellitus para cada sexo, en determinados departamentos y años. Se aplicó el Modelo de Poisson discreto, asumiendo que los casos seguían esa distribución.

La aplicación novedosa de esta técnica permite testear si la distribución de un determinado evento de salud en el espacio y el tiempo se produce por azar; esto se realiza mediante un rastreo gradual de una ventana a través del tiempo y el espacio, señalando el número de casos observados y esperados dentro de la ventana, en cada localización. Bajo la hipótesis nula, el número esperado de casos en cada 
área es proporcional a su tamaño poblacional, o a las personas-año en esa área. Los casos observados son las defunciones ingresadas al programa 28.

La ventana de exploración para este caso es un cilindro de radios variables, cuya base representa el área del posible conglomerado y la altura representa el tiempo de duración del mismo; la ventana con la máxima verosimilitud (likelihood ratio) constituye el conglomerado más probable, es decir, el conglomerado con menor probabilidad de explicarse por azar, cuyo valor de p es asignado por el Test de hipótesis de Montecarlo, a un nivel del 95\% de significancia. Se realizaron 9.999 replicaciones de Montecarlo.

El límite máximo fijado para la ventana espacial fue del 50\% de la población en riesgo, mientras que el límite máximo fijado para la ventana temporal fue del 50\% del tiempo abarcado por el período 1990-2012.

Además de la identificación de conglomerados, el programa calcula el riesgo relativo y la tasa anual de mortalidad. El riesgo relativo es el resultado del cociente entre el riesgo estimado dentro del conglomerado y el riesgo estimado fuera del mismo, los cuales derivan a su vez del cociente entre los casos observados y esperados dentro o fuera del agregado, respectivamente.

\section{Resultados}

El conglomerado primario más probable de alta mortalidad prematura por diabetes mellitus, tanto en varones como en mujeres, se ubicó en la región centro-norte de Argentina, con epicentro en el departamento de Punilla de la Provincia de Córdoba en ambos casos (Figuras 1 y 2); los períodos identificados correspondieron a 1998-2008 para los varones y 1997-2007 para las mujeres, con riesgos relativos estadísticamente significativos cercanos al doble, con respecto al resto de Argentina en ambos sexos (Tabla 1). En los varones se registraron 11.603 casos en el período, cuando se esperaban 6.866; en las mujeres la relación fue 14.196 y 8.800 respectivamente (Tabla 1).

Los conglomerados identificados de alta mortalidad esperada por diabetes mellitus tuvieron una localización geográfica similar a los de la mortalidad prematura: los epicentros del conglomerado primario se localizaron en los departamentos de Los Andes (Provincia de Salta) para las mujeres (Figura 3) y Santa Rosa (Provincia de Mendoza) para los varones (Figura 4). Así, la alta mortalidad prematura por diabetes mellitus tuvo su epicentro tanto para los varones, como para las mujeres, en la Provincia de Córdoba y cubrió el centro-norte de la Argentina, mientras que las regiones de alta mortalidad esperada presentaron variaciones espacio-temporales entre mujeres y varones, con las primeras mostrando un retraso temporal de dos años y un desplazamiento espacial hacia el norte de Argentina. En comparación con los conglomerados espacio-temporales de mortalidad prematura, los conglomerados de mortalidad esperada cubrieron un área más extensa en ambos sexos.

Se identificó un conglomerado primario de baja mortalidad prematura por diabetes mellitus para ambos sexos, con epicentro en los partidos de General Juan de Madariaga (mujeres) y General Lavalle (varones), ocupando el este de la Provincia de Buenos Aires, en el período 2002-2012 (Figuras 1 y 2), con riesgos relativos estadísticamente significativos de alrededor del $50 \%$ inferiores con respecto al resto de la Argentina (Tabla 1). Adicionalmente, se registró otro conglomerado de baja mortalidad prematura en mujeres, localizado en la parte sur de las provincias de Buenos Aires y La Pampa y gran parte de la región Patagónica, durante el período 1990-2000 (Figura 1; Tabla 1).

En el caso de la concentración espacio-temporal de mortalidad esperada baja en varones, se detectó un conglomerado en gran parte del Gran Buenos Aires, con epicentro en el Partido de Lanús, durante el período 2002-2012 (Figura 4; Tabla 1). Con respecto a las mujeres, el conglomerado de mortalidad esperada baja se localizó en la mitad este de la Provincia de Buenos Aires (Figura 3), con epicentro en el partido de General Juan de Madariaga, durante el período 2002-2012 (Tabla 1).

\section{Discusión}

Este estudio demostró la existencia de interacción espacio-temporal en la mortalidad por diabetes mellitus tanto en población femenina, como población masculina. Por lo tanto, el contexto geográfico 
Figura 1

Conglomerados espacio-temporales de baja y alta mortalidad prematura por diabetes mellitus en población femenina. Argentina, 1990-2012.

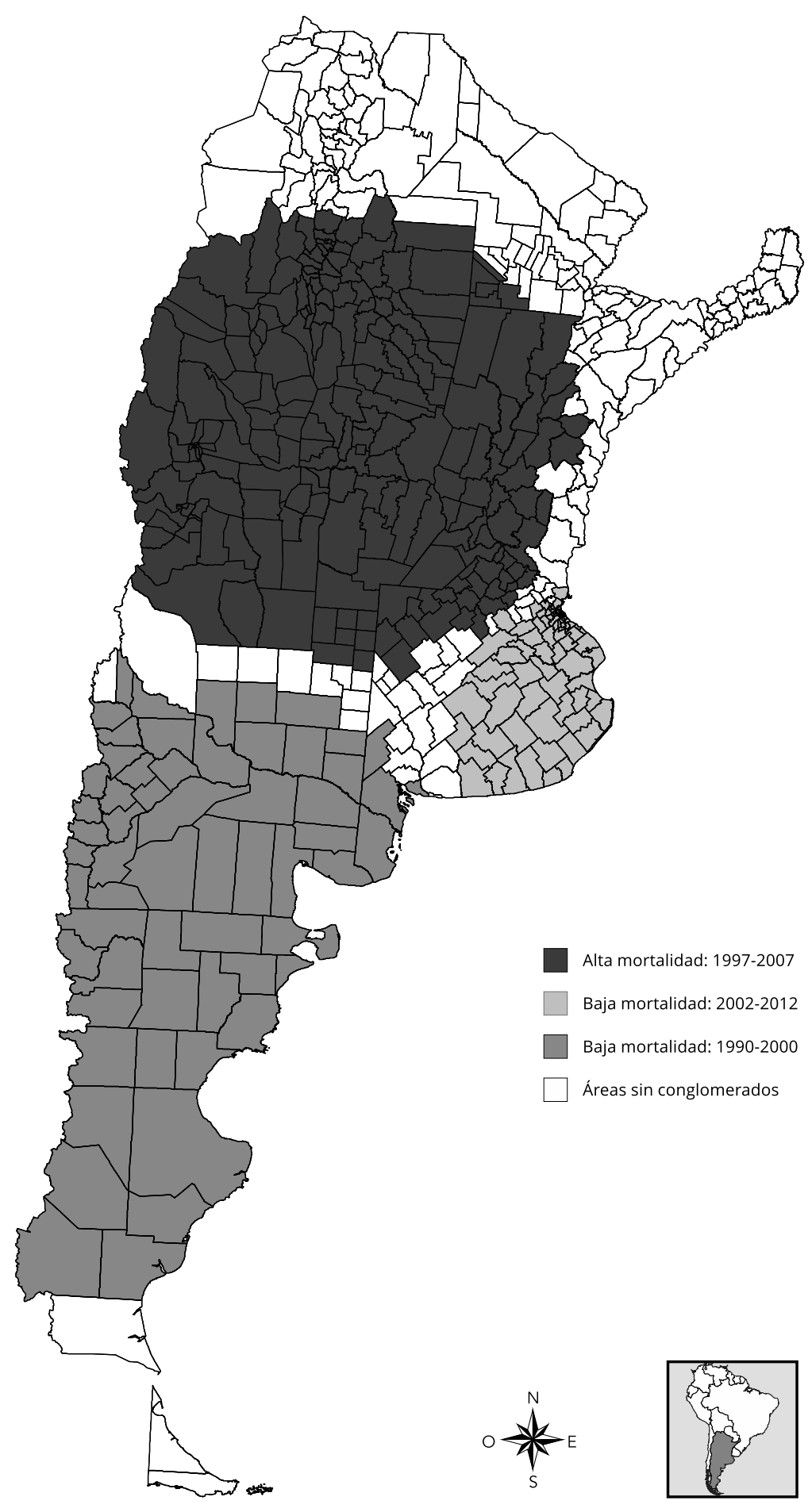




\section{Figura 2}

Conglomerados espacio-temporales de baja y alta mortalidad prematura por diabetes mellitus en población masculina. Argentina, 1990-2012.

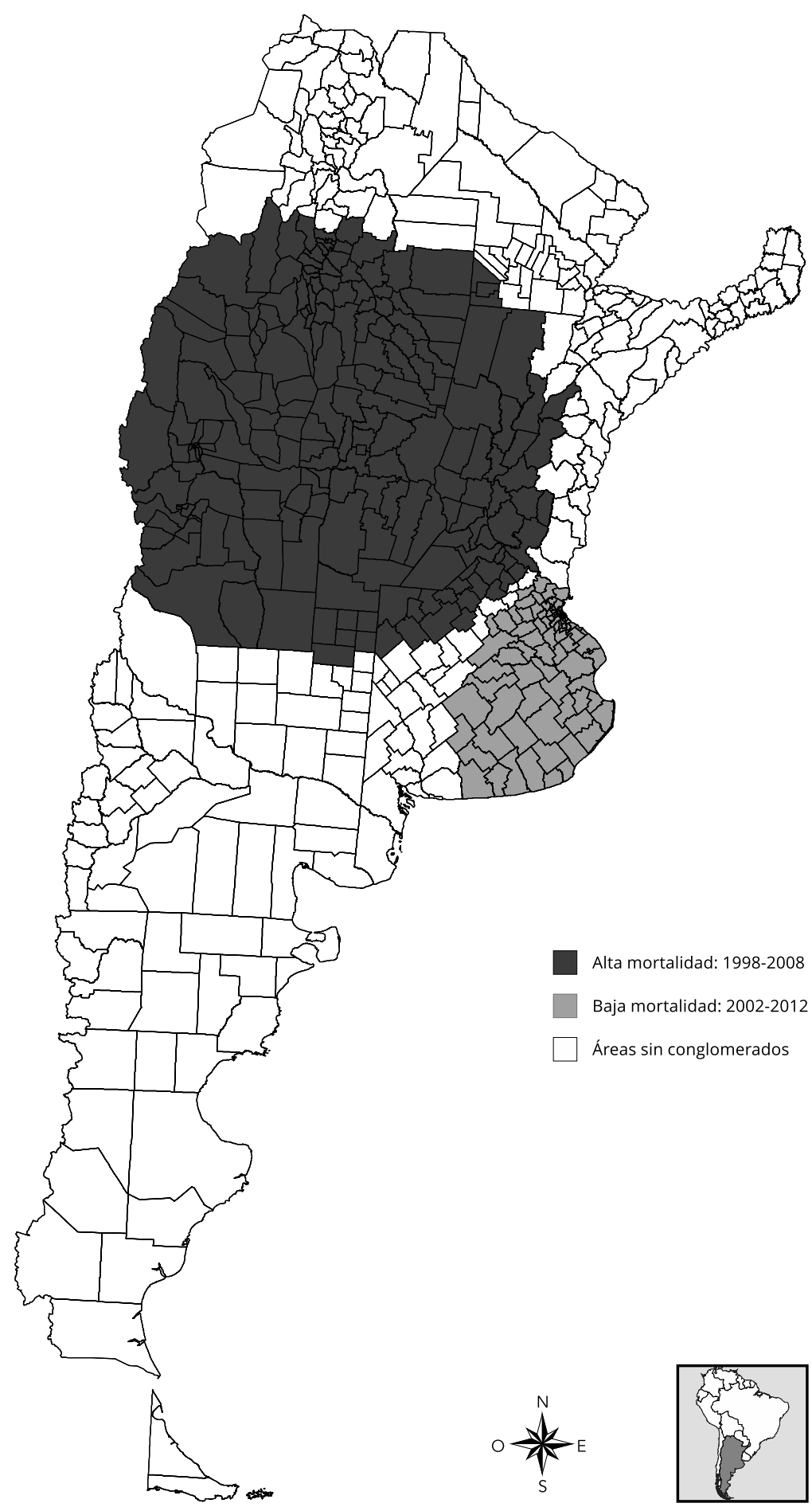




\section{Tabla 1}

Conglomerados espacio-temporales de alta y baja mortalidad por diabetes mellitus: epicentro geográfico; período temporal; casos observados y esperados, tasa anual de mortalidad y riesgo relativo según sexo. Argentina, 1990-2012.

\begin{tabular}{|c|c|c|c|c|c|c|}
\hline & Período & $\begin{array}{c}\text { Casos } \\
\text { observados }\end{array}$ & $\begin{array}{c}\text { Casos } \\
\text { esperados }\end{array}$ & $\begin{array}{c}\text { Tasa anual } \\
*\end{array}$ & $\begin{array}{l}\text { Riesgo } \\
\text { relativo }\end{array}$ & $\begin{array}{c}\text { Valor de } \\
\qquad p^{* *}\end{array}$ \\
\hline \multicolumn{7}{|l|}{ Alta mortalidad } \\
\hline \multicolumn{7}{|l|}{ Prematura mujeres } \\
\hline Conglomerado 1: Córdoba *** & 1997-2007 & 14.196 & 8.800 & 51,9 & 1,85 & $<0,001$ \\
\hline \multicolumn{7}{|l|}{ Prematura varones } \\
\hline Conglomerado 1: Córdoba *** & $1998-2008$ & 11.603 & 6.866 & 51,5 & 1,96 & $<0,001$ \\
\hline \multicolumn{7}{|l|}{ Esperada mujeres } \\
\hline Conglomerado 1: Salta *** & $1995-2005$ & 11.402 & 7.794 & 246,3 & 1,67 & $<0,001$ \\
\hline \multicolumn{7}{|l|}{ Esperada varones } \\
\hline Conglomerado 1: Mendoza *** & $1997-2007$ & 13.541 & 9.060 & 308,3 & 1,69 & $<0,001$ \\
\hline \multicolumn{7}{|l|}{ Baja mortalidad } \\
\hline \multicolumn{7}{|l|}{ Prematura mujeres } \\
\hline Conglomerado 1: Buenos Aires *** & $2002-2012$ & 7.116 & 11.352 & 20,2 & 0,57 & $<0,001$ \\
\hline Conglomerado 2 : Chubut $* \star \star$ & $1990-2000$ & 407 & 871 & 15,0 & 0,46 & $<0,001$ \\
\hline \multicolumn{7}{|l|}{ Prematura varones } \\
\hline Conglomerado 1: Buenos Aires *** & $2002-2012$ & 5981 & 9.282 & 19,7 & 0,58 & $<0,001$ \\
\hline \multicolumn{7}{|l|}{ Esperada mujeres } \\
\hline Conglomerado 1: Buenos Aires *** & $2002-2012$ & 5274 & 9.650 & 92,0 & 0,47 & $<0,001$ \\
\hline \multicolumn{7}{|l|}{ Esperada varones } \\
\hline Conglomerado 1: Buenos Aires *** & $2002-2012$ & 3901 & 6.834 & 117,8 & 0,53 & $<0,001$ \\
\hline
\end{tabular}

Nota: Conglomerado 1 = conglomerado primario; Conglomerado 2 = conglomerado secundario.

* Por 100.000 habitantes;

** Nivel del 95\% de significancia;

*** Provincia que contiene el departamento epicentro del conglomerado.

y su tendencia temporal tienen importancia al analizar la mortalidad por diabetes mellitus. Este contexto parece afectar de manera similar a la mortalidad prematura por diabetes mellitus en hombres y mujeres, ya que no se encontraron grandes diferencias en la distribución espacio-temporal de los conglomerados de alta mortalidad. En un estudio llevado a cabo en Taiwán tampoco se observaron diferencias en la concentración espacial de la mortalidad por diabetes mellitus en ambos sexos 18 . En Portugal, se registró un doble patrón geográfico de aumento de la mortalidad por diabetes mellitus en sentido litoral-interior y norte-sur para ambos sexos, durante los últimos años del período 1989-2010 29. Sin embargo, al analizar las diferencias entre conglomerados de población femenina y masculina, considerando muertes esperadas, se observaron diferencias espacio-temporales entre los dos conglomerados registrados. El conglomerado de población femenina ocupó, a diferencia de la población masculina, las provincias del extremo norte argentino, con un menor nivel de desarrollo económico en comparación com el resto del país. Por lo tanto, se hipotetiza que la mortalidad esperada por diabetes mellitus pudo estar más relacionada a desigualdades territoriales, en el caso de las mujeres, con respecto a los hombres. En relación con esto, se ha encontrado que las desigualdades socioeconómicas en la mortalidad por diabetes mellitus son más pronunciadas en mujeres que en hombres 30 .

Investigaciones realizadas en Estados Unidos que han estudiado la distribución geográfica de la enfermedad por regiones, han encontrado un peso desproporcionado de las tasas de incidencia o mortalidad por diabetes mellitus entre las minorías étnicas 17,22,31,32. Otros trabajos que se han concentrado en el nivel local han generado información espacial útil para analizar la distribución de la diabetes mellitus en la población y han encontrado un mayor peso de la morbilidad y mortalidad por 


\section{Figura 3}

Conglomerados espacio-temporales de baja y alta mortalidad esperada por diabetes mellitus en población femenina. Argentina, 1990-2012.

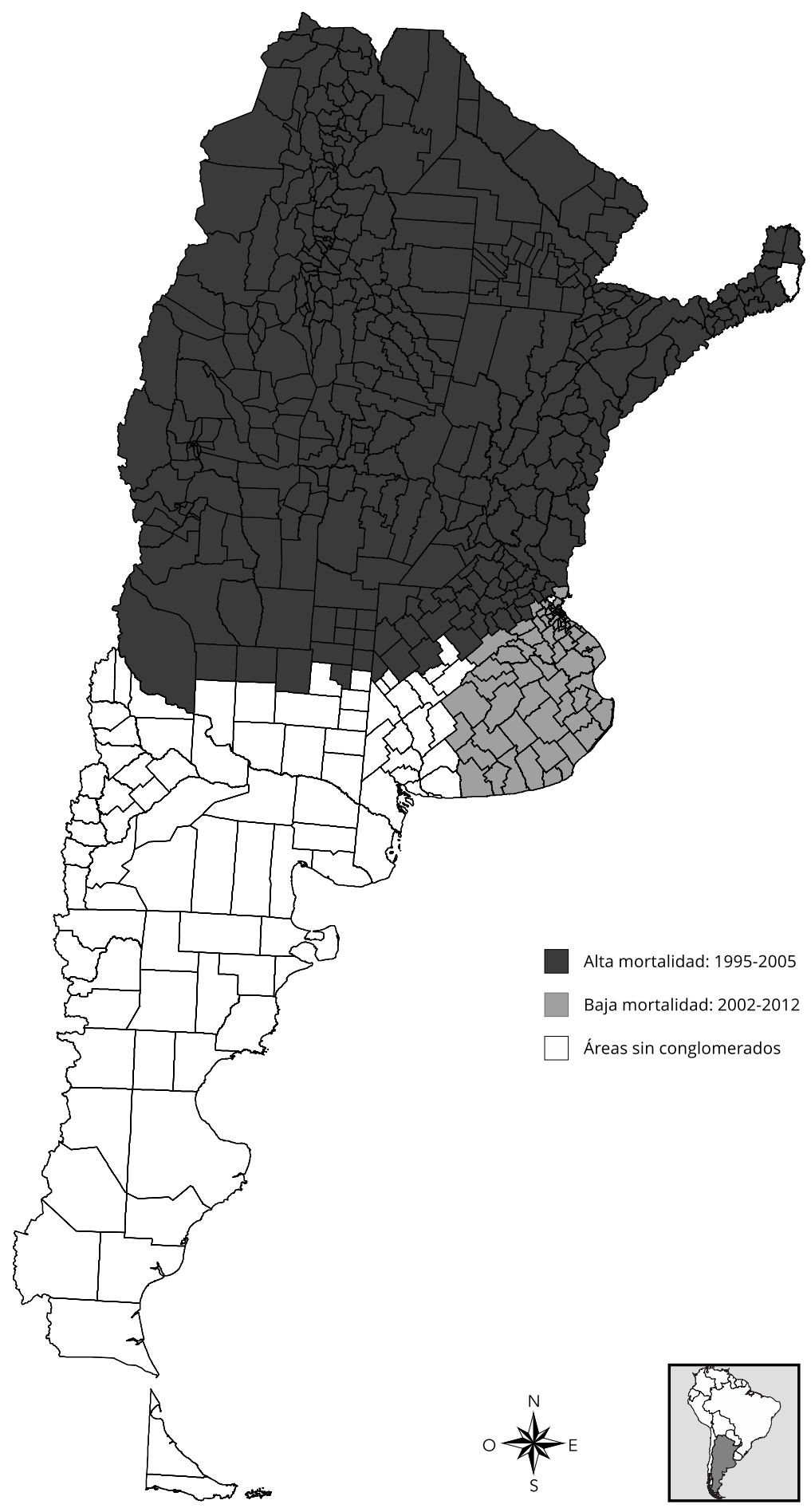




\section{Figura 4}

Conglomerados espacio-temporales de baja y alta mortalidad esperada por diabetes mellitus en población masculina. Argentina, 1990-2012.

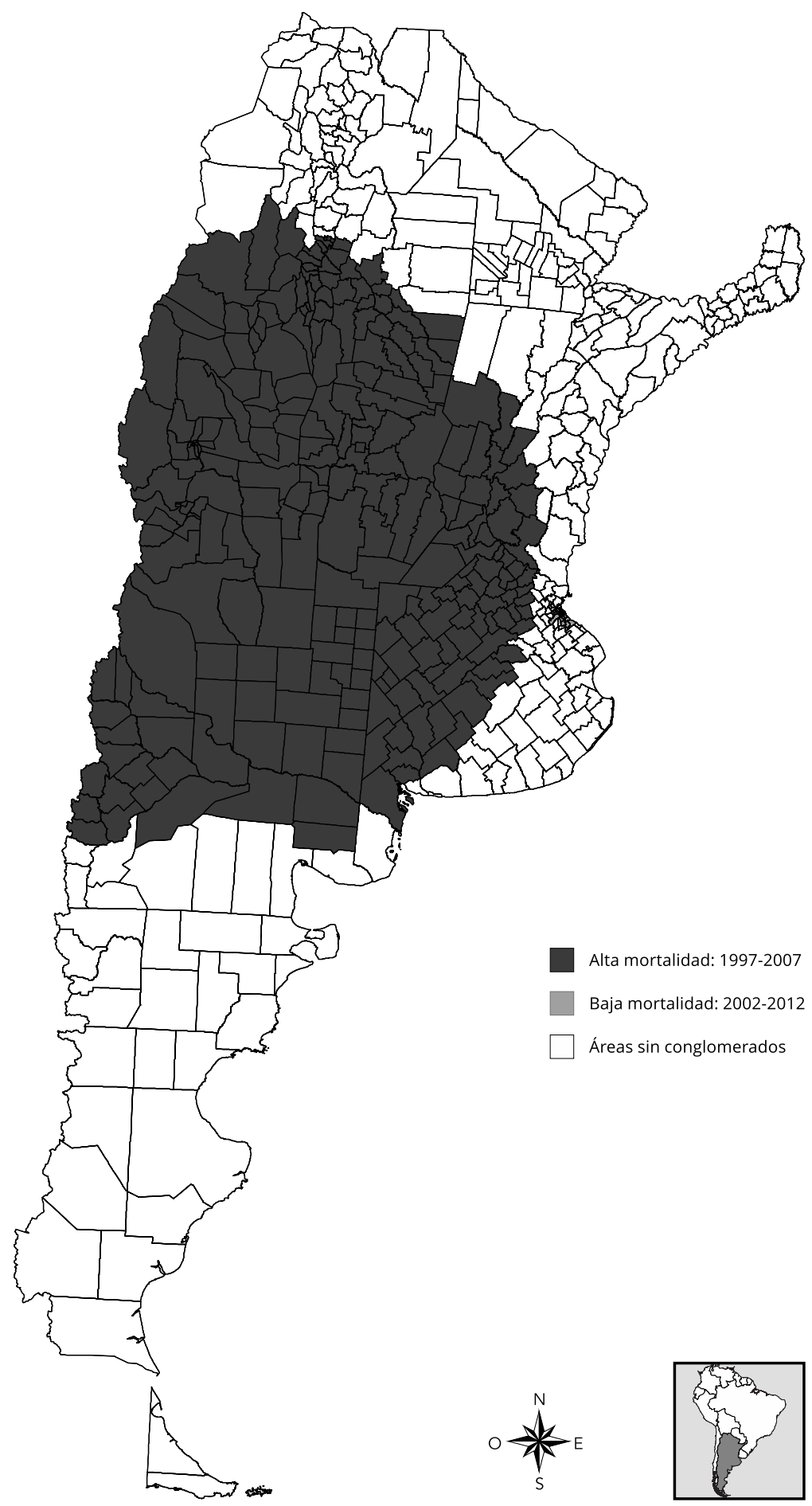


diabetes mellitus sobre las poblaciones vulnerables de acuerdo con sus características socioeconómicas 19,20,33,34,35. Salvo en el caso del conglomerado espacio-temporal de alta mortalidad esperada en mujeres mencionado anteriormente, en los cuales parte de su localización ocupó las provincias del norte argentino, los restantes conglomerados de alta mortalidad no se localizaron principalmente en estas provincias. Excepto el conglomerado de alta mortalidad esperada en mujeres, los restantes conglomerados de alta mortalidad, tanto esperada como prematura, se localizaron en el centro-norte de la Argentina, pero sin ocupar las provincias del extremo norte del país. Al analizar los datos de las encuestas nacionales de factores de riesgo, se pueden observar aumentos significativos de la prevalencia de diabetes mellitus en las regiones de Cuyo y el Noroeste argentino entre los años 2005 y 2009 36. Gran parte de las provincias que conforman ambas regiones (Catamarca, La Rioja, Mendoza, San Luís, San Juan y Santiago del Estero) ocuparon los conglomerados de alta mortalidad registrados en este estudio.

Las características que asume la mortalidad por diabetes mellitus en Argentina contribuyen a delinear el comportamiento de la enfermedad en nuestra población, sobre la cual el sistema de salud puede intervenir con la implementación de políticas sanitarias para la prevención y el control. El acceso a los servicios de atención de diabetes mellitus, la cobertura de las prestaciones y su impacto en la prolongación de la vida resultan claves en el análisis de las condiciones que influyen en el retraso de la mortalidad en una enfermedad de perfil crónico.

La diabetes mellitus es una ECNT, que requiere de tratamiento precoz y adecuado para evitar complicaciones micro y macro-vasculares. El control inadecuado de la enfermedad y los factores de riesgo cardiovascular asociados generan un incremento de la morbi-mortalidad y de su carga socio-económica. Entre ellos se incluye el diagnóstico tardío, la denominada inercia prescriptiva, especialmente de insulina, y la educación insuficiente de profesionales de salud y pacientes. Estudios realizados en Argentina aseguran que la diabetes mellitus 2 se diagnostica tardíamente, con una eficacia del 50\% en el reconocimiento y tratamiento de los pacientes. A su vez, menos de la mitad de ellos alcanzaría las metas terapéuticas: se calcula que el 66\% de los pacientes en tratamiento desarrollan complicaciones crónicas 37.

Respecto de la mortalidad prematura, es probable que su ocurrencia sume a los factores que propician focos de alta mortalidad esperada, agravantes relacionados con deficiencias en la atención o inaccesibilidad a los servicios de salud. En cuanto a la disminución de la mortalidad prematura, en este estudio se encontraron dos conglomerados espacio-temporales de baja mortalidad: en la mitad este de la provincia de Buenos Aires, durante el período 2002-2012 (ambos sexos), y en gran parte de la región patagónica, entre los años 1990 y 2000 (sólo en mujeres). En este conglomerado patagónico, la falta de datos relacionados con factores de riesgo y distribución de tratamientos de la diabetes mellitus no nos permite plantear hipótesis acerca de la disminución significativa de muertes prematuras en la población femenina de esta región durante la década del noventa. Con respecto al primer conglomerado, la creación en la Provincia de Buenos Aires de un Programa de Prevención, Atención y Tratamiento del Paciente Diabético (PRODIABA), a partir de 1996, pudo haber jugado un rol positivo en el acceso al tratamiento de la diabetes mellitus en personas sin cobertura de salud y sin recursos económicos para obtenerlo. Entre los años 2002 y 2006 hubo un aumento de casi el 35\% en el número de pacientes bajo el PRODIABA 38. A esto puede agregarse la incorporación en 2003 del Programa Remediar a la Política Nacional de Medicamentos, con el propósito de asegurar el acceso a medicamentos en el sistema público de salud y los objetivos de fortalecer el primer nivel de atención y el uso racional de medicamentos, que contribuyó con la cobertura farmacológica de la diabetes mellitus, no sólo en la Provincia de Buenos Aires, sino en el resto de Argentina 39. Además, las encuestas nacionales de factores de riesgo aplicadas en 2005, 2009 y 2013 mostraron una tendencia creciente de la frecuencia de prácticas preventivas en control de glucemia, colesterol y presión arterial en la región pampeana y el Gran Buenos Aires 36,40.

Estas políticas de salud se llevaron a cabo en un contexto de creciente desarrollo de iniciativas sanitarias enfocadas en la prevención de las enfermedades no transmisibles. Los hábitos saludables, la alimentación sana, la actividad física y la cesación tabáquica ganaron terreno dentro de las políticas nacionales de salud 41 .

La sanción de la ley nacional de diabetes en 1989 (Ley Nacional 23.753) 42, así como la institucionalización de programas específicos pueden considerarse antecedentes de este proceso; en el año 
2013 se sancionó una nueva ley de diabetes (Ley Nacional 26.914) 43 que amplía la cobertura de las prestaciones previstas por la Ley Nacional 23.573. El Programa Nacional de Diabetes (PRONADIA) comienza a funcionar en 1999, después de la reglamentación de la ley nacional de diabetes (Decreto 1.271) 44. Entre 1990 y 2010, todas las provincias suscriben la ley nacional de diabetes, y se generan programas provinciales para la provisión de medicamentos y reactivos de diagnóstico, la prevención de la enfermedad, el diagnóstico precoz, y la producción de información estadística. Algunos también avanzan sobre la prevención de las enfermedades cardiovasculares y la promoción de la actividad física y la alimentación saludable.

Tomando en cuenta la dimensión temporal de este estudio, los resultados demostraron que los conglomerados de alta mortalidad por diabetes mellitus, en sus modalidades prematura y esperada, ocupan un período que va de 1995-1998 a 2005-2008. En cambio, los conglomerados de baja mortalidad, también en sus variantes prematura y esperada, se desplazan mayoritariamente hacia después de los años 2000, en los años que van de 2002-2012.

Estas variaciones en la ocurrencia de conglomerados pudieron estar relacionadas con el fortalecimiento de las iniciativas nacionales y provinciales explicado anteriormente, en el marco de la recuperación social y económica del país, a partir de la primera década de 2000, enfocadas en la prevención de las enfermedades no transmisibles y la cobertura de los tratamientos en diabetes mellitus, factores que parecen haber tenido un impacto positivo en la disminución de la morbi-mortalidad en la Argentina.

Según los datos de la escuesta nacional de factores de riesgo también se observó, para el total nacional, un incremento en la frecuencia de prácticas preventivas ligadas a factores de riesgo, como el control de la presión arterial, glucemia y colesterol entre los años 2005 y 2013 36,40. Esta mayor cobertura pudo haber influenciado un acceso más temprano al diagnóstico de la diabetes mellitus en este período que, sumado a una mayor cobertura farmacológica de la diabetes mellitus, se relacionó a la no ocurrencia de conglomerados de alta mortalidad durante los últimos años del período 1990-2012. Análisis posteriores en profundidad contribuirán con despejar los determinantes de las diferencias en su distribución regional.

La relación temporal entre momentos de crisis económicas, políticas y sociales y los indicadores de mortalidad por diabetes mellitus en poblaciones ha sido documentada por algunos estudios recientes arrojando resultados dispares 45,46, observación que da cuenta de la complejidad de las múltiples dimensiones intervinientes. Los resultados de la presente investigación también permiten evidenciar una disminución del riesgo de muerte por diabetes mellitus con la recuperación institucional del país después de la crisis social y económica que atravesó Argentina hacia finales de 2001.

El análisis realizado en este trabajo presentó algunas limitaciones. En primer lugar, la falta de datos de mortalidad durante el primer quinquenio en diez provincias argentinas no permitió detectar con mayor precisión conglomerados espacio-temporales durante ese período. En segundo lugar, la falta de datos referidos a proyecciones poblacionales por departamento, desagregados por sexo y grupos de edad, y el uso de grupos quinquenales de población total nos impidieron calcular denominadores poblacionales de manera más precisa.

En este trabajo se demostró que la mortalidad por diabetes mellitus experimentó variaciones espacio-temporales a lo largo de poco más de las dos últimas décadas en la Argentina. En general, se observaron similitudes en la distribución de los conglomerados espacio-temporales de alta mortalidad en ambos sexos, con una concentración espacial más acotada en el caso de la mortalidad prematura por diabetes mellitus. En cambio, en el caso de los conglomerados de baja mortalidad se registraron áreas más extensas de baja mortalidad prematura, con respecto a los conglomerados de mortalidad esperada, principalmente en el este de la Provincia de Buenos Aires durante la última década del período 1990-2012.

Por último, cabe destacar que la diabetes mellitus constituye una temática en desarrollo en nuestro país, por lo que no cuenta con la proliferación de estudios que se observan en otras áreas, como es el caso de las enfermedades cardiovasculares. La presente investigación constituye el primer abordaje espaciotemporal de la diabetes mellitus con nivel de agregación subnacional en Argentina. Surgen, a partir de los resultados, múltiples interrogantes que merecerían afrontarse con futuras investigaciones que pudieran incorporar factores determinantes de la muerte por diabetes mellitus, así como aquellos relacionados con la distribución diferencial del acceso a los servicios de salud en el país. 


\section{Colaboradores}

C. M. Leveau, M. J. Marro, V. Alonso y A. E. B. Lawrynowicz participaron en la elaboración de la idea central, del texto y en la revisión final.

\section{Agradecimientos}

La investigación se desarrolló en el Instituto Nacional de Epidemiología “Dr. Juan H. Jara”, dependiente de la Administración Nacional de Laboratorios e Institutos de Salud del Ministerio de Salud de Argentina, durante el año 2014. Resultados preliminares fueron presentados en las Primeras Jornadas Nacionales de Salud Pública, organizadas por estas instituciones en Mar del Plata, Provincia de Buenos Aires, en octubre de 2014. Carlos Marcelo Leveau agradece a Betiana Menestrina por el aporte bibliográfico.

\section{Referencias}

1. Asociación Latinoamericana de Diabetes. Guías ALAD de Diagnóstico, control y tratamiento de la diabetes mellitus tipo 2, 2006. http://www.alad latinoamerica.org/phocadownload/guias\%20alad. pdf (accedido el 04/Abr/2014)

2. International Diabetes Federation. Diabetes atlas. http://www.idf.org/sites/default/files/EN_6E_ Atlas_Full_0.pdf (accedido el 22/Abr/2015).

3. Ministerio de Salud de la Nación Argentina. Estadísticas vitales. Información básica año 2012 http://www.deis.gov.ar/Publicaciones/Archivos/ Serie5Nro56.pdf (accedido el 10/Sep/2014).
4. Ministerio de Salud de la Nación Argentina. Evolución de la mortalidad según causas. República Argentina período 1997-2006. http://www.deis. gov.ar/publicaciones/Archivos/Serie12nro6.pdf (accedido el 06/May/2015)

5. Organización Panamericana de la Salud. Técnicas para la medición del impacto de la mortalidad: Años Potenciales de Vida Perdidos. Boletín Epidemiológico 2003; 24:1-4. http://cidbimena.desas tres.hn/docum/ops/publicaciones/Epidemiologi co/EB_v24n2.pdf (accedido el 14/Dic/2015). 
6. Murray CJL, Ezzati M, Flaxman AD, Lim S, Lozano R, Michaud C, et al. GBD 2010: design, definitions, and metrics. Lancet 2012; 380:2063-6.

7. Tobler W. A computer movie simulating urban growth in the Detroit region. Econ Geogr 1970; 46:234-40.

8. Buzai GD, Baxendale CA. Análisis socioespacial con Sistemas de Información Geográfica: perspectiva científica, temáticas de bases raster. Buenos Aires: Lugar Editorial; 2011.

9. Peuquet DJ. It's about time: a conceptual framework for the representation of temporal dynamics in geographic information systems. Ann Assoc Am Geogr 1994; 84:441-61.

10. Werneck GL, Struchiner CJ. Estudos de agregados de doença no espaço-tempo: conceitos, técnicas e desafíos. Cad Saúde Pública 1997; 13:611-24.

11. Pike MC, Smith PG. Disease clustering: a generalization of knox's approach to the detection of space-time interactions. Biometrics 1968; 24 541-56.

12. Rytkonen M, Ranta J, Tuomilehto J, Karvonen M. Bayesian analysis of geographical variation in the incidence of type I diabetes in Finland. Diabetologia 2001; 44 Suppl 3:B37-44.

13. Samuelsson U, Löfman O. Geographical mapping of type 1 diabetes in children and adolescents in south east Sweden. J Epidemiol Community Health 2004; 58:388-92.

14. Cardwell CR, Carson DJ, Patterson CC. Higher incidence of childhood-onset type 1 diabetes mellitus in remote areas: a UK regional small-area analysis. Diabetologia 2006; 49:2074-7.

15. Geir A, Lars CS, Pal RN, Oddmund S, Geir J. Spatiotemporal trends and age-period-cohort modeling of de incidence of type 1 diabetes among children ages < 15 years in Norway 1973-1982 and 1989-2003. Diabetes Care 2007; 30:884-9.

16. Torres Avilés F, Carrasco E, Icaza G, Pérez-Bravo F. Clustering of cases of type 1 diabetes in high socioeconomic communes in Santiago de Chile: spatio-temporal and geographical analysis. Acta Diabetol 2010; 47:251-7.

17. Liese AD, Lawson A, Song HR, Hibbert JD, Porter $\mathrm{DE}$, Nichols M, et al. Evaluating geographic variation in type 1 and type 2 diabetes mellitus incidence in youth in four US regions. Health Place 2010; 16:547-56.

18. Tsai P-J, Lin M-L, Chu Ch-M, Perng Ch-H. Spatial autocorrelation analysis of health care hotspots in Taiwan in 2006. BMC Public Health 2009; 9:464.

19. Green C, Hoppa RD, Young TK, Blanchard JF. Geographic analysis of diabetes prevalence in an urban area. Soc Sci Med 2003; 57:551-60.
20. Campos FG, Barrozo LV, Ruiz T, César CLG, Barros MBA, Carandina L, et al. Distribuição espacial dos idosos de um município de médio porte do interior paulista segundo algumas características sócio-demográficas e de morbidade. Cad Saúde Publica 2009; 25:77-86.

21. Institute for Health Metric and Evaluation. Cause of Death (COD) visualization. http://vizhub. healthdata.org/cod/ (accedido el 30/Dic/2015).

22. Barker LE, Kirtland KA, Gregg EW, Geiss LS, Thompson TJ. Geographic distribution of diagnosed diabetes in the U.S.A. diabetes belt. Am J Prev Med 2011; 40:434-9.

23. Equivalencia de la lista reducida de causas de muerte CIE-10 con la CIE-9. http://cie9.com/De funciones_Equivalencia_cie10_y_cie9.pdf (accedido el 06/Abr/2015).

24. Ministerio de Salud y Acción Social de la Nación Argentina. Estadísticas vitales - información básica Año 1994. Buenos Aires: Ministerio de Salud y Acción Social; 1994.

25. Page A, Tobias M, Glover J, Wright C, Hetzel D, Fisher E. Australian and New Zealand atlas of avoidable mortality. Adelaide: Public Health Information Development Unit, University of Adelaide; 2006.

26. Wheisz D, Gusmano MK, Rodwin VG, Neuberg LG. Population health and health system: a comparative analysis of avoidable mortality in three nations and their world cities. Eur J Public Health 2008; 18:166-72.

27. Wheller L, Baker A, Griffiths C, Rooney C. Trends in avoidable mortality in England and Wales, 1993-2005. Health Stat Q 2007; 34:6-25.

28. Kulldorf M. SaTScan user guide, for versión 9.3. http://www.satscan.org/techdoc.html (accedido el 21/Jun/2014).

29. Santana P, Costa C, Loureiro A, Raposo J, Boavida JM. Geografias da diabetes mellitus em Portugal: como as condições do contexto influenciam o risco de morrer. Acta Med Port 2014; 27:309-17. 
30. Espelt A, Borrell C, Roskam AJ, Rodríguez-Sanz M, Stirbu I, Dalmau-Bueno A, et al. Socioeconomic inequalities in diabetes mellitus across Europe at the beginning of the 21st century. Diabetologia 2008; 51:1971-9.

31. American Indian, Hispanic, and Non-Hispanic white populations, 1958-1987. Diabetes Care 1993; 16:306-9.

32. Gilliland F, Owen C, Gilliland S, Carter J. Temporal trends in diabetes mortality among American Indians and Hispanics in New Mexico: birth cohort and period effects. Am J Epidemiol 1997; 145:422-31.

33. Curtis A, Lee WA. Spatial patterns of diabetes related health problems for vulnerable populations in Los Angeles. Int J Health Geogr 2010; 9:43.

34. Santana Castañeda G, Santana Juárez MV, Némiga XA, Campos Alanís J. Metodología para determinar la distribución espacial y tendencia de la diabetes mellitus, utilizando sistemas de información geográfica sobre análisis cluster. Facultad de Geografía, Universidad Autónoma del Estado de México. http://www.inegi.org.mx/eventos/2011/ Conf_Ibero/doc/ET3_28_SANTANA.pdf (accedido el 14/Dic/2015)

35. Tompkins J, Luginaah I, Booth GL, Harris SB. The geography of diabetes in London, Canada: the need for local level policy for prevention and management. Int J Environ Res Public Health 2010; 7:2407-22.

36. Ministerio de Salud de la Nación Argentina. 3a Encuesta Nacional de Factores de Riesgo para Enfermedades no Transmisibles. Buenos Aires: Ministerio de Salud de la Nación Argentina/Instituto Nacional de Estadística y Censos; 2015.

37. Gagliardino J, Costa Gil J, Faingold M, Litwak L, Fuente G. Insulina y control de la diabetes en la Argentina. Medicina 2013; 73:520-8.

38. Orchuela J. Acceso y Programas de suministro de medicamentos y vacunas del Ministerio de Salud de la provincia de Buenos Aires desde los años 90. V Jornadas de Sociología de la UNLP. http:// www.aacademica.org/000-096/255.pdf (accedido el 13/Ene/2016).

39. Ministerio de Salud de la Nación. Programa Remediar. http://www.remediar.gov.ar (accedido el 06/Jun/2015).
40. Ferrante D, Linetzky B, Konfino J, King A, Laspiur S. Encuesta Nacional de Factores de Riesgo 2009: evolución de la epidemia de enfermedades crónicas no transmisibles en Argentina. Estudio de corte transversal. Rev Argent Salud Pública 2011; 2:34-41.

41. Lawrynowicz A, Alonso V. El tratamiento de la diabetes mellitus en la normativa sanitaria argentina: una sistematización. Rev Argent Salud Pública 2013; 4:39-44.

42. Presidencia de la Nación. Ley Nacional 23.753 de 29 de septiembre de 1989. Boletín Oficial de la República Argentina 1989; 6 Oct.

43. Presidencia de la Nación. Ley Nacional 26.914 (modificatoria de la Ley Ley 23.753) de 27 de noviembre de 2013. Boletín Oficial de la República Argentina 2013; 17 dec.

44. Poder Ejecutivo Nacional. Decreto 1.271 de 23 de octobre de 1998. Decreto Reglamentario de la Ley 23.753, sobre Problematica y Prevencion de la Diabetes. Boletín Oficial de la República Argentina 1998; 2 nov.

45. Borowy I. Similar but different: health and economic crisis in 1990s Cuba and Russia. Soc Sci Med 2011; 72:1489-98.

46. Franco M, Orduñez P, Caballero B, Tapia Granados JA, Lazo M, Bernal JL, et al. Impact of energy intake, physical activity, and population-wide weight loss on cardiovascular disease and diabetes mortality in Cuba, 1980-2005. Am J Epidemiol 2007; 166:1474-80. 


\section{Abstract}

The aim of this study was to identify spatialtemporal clusters of high and low diabetes-related mortality from 1990 to 2012 in Argentina. This was a spatial-temporal retrospective ecological study in the population older than 34 years living in Argentina, according to sex, from 1990 to 2012. The spatial units of analysis consisted of the country's departments (subdivisions of the provinces) plus the Autonomous City of Buenos Aires. Spatial-temporal exploration technique was used to detect clusters with high and low mortality. Areas with high mortality from diabetes mellitus were found in the Central-West of the country, and areas with low mortality were found in the coastal region of the province of Buenos Aires and Patagonia. Clusters with high mortality occupied a period from 1995 to 2008; clusters with low mortality shifted towards the years 2002 to 2012. The recent drop in mortality from diabetes was not geographically homogeneous, but displayed a marked decrease in the eastern area of the Province of Buenos Aires and the Autonomous City of Buenos Aires.

Diabetes Mellitus; Premature Mortality; Cluster Analysis

\section{Resumo}

O objetivo desta pesquisa foi identificar conglomerados espaço-temporais de alta e baixa mortalidade por diabetes mellitus durante o periodo 1990-2012 na Argentina. Foi realizado um estudo ecológico retrospectivo espaço-temporal, considerando a população maior de 34 anos, residente na Argentina, segundo o sexo, durante o período de 1990-2012. As unidades espaciais de análises foram constituídas pelos departamentos, junto com a Cidade Autônoma de Buenos Aires. Foi empregada a técnica de exploração espaço-temporal para a detecção de conglomerados de alta e baixa mortalidade. Foram encontradas áreas de elevada mortalidade por diabetes mellitus no Centro-oeste do país, e zonas de baixa mortalidade na região costeira da província de Buenos Aires e Patagônia. Os conglomerados de alta mortalidade ocuparam um período compreendido entre 1995 e 2008; os de baixa mortalidade foram no período de 2002-2012. A diminuição recente na mortalidade por diabetes mellitus não foi geograficamente homogênea, com destaque para o leste da província de Buenos Aires e a Cidade Autônoma de Buenos Aires.

Diabetes Mellitus; Mortalidade Prematura; Análise por Conglomerados
Recibido el 15/Oct/2015

Versión final presentada el 15/Ene/20216 Aprobado el 06/Abr/2016 